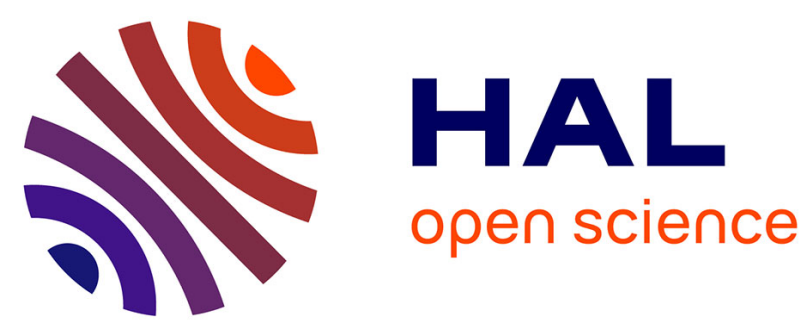

\title{
Improving the resolution of pulsed thermal wave images with a simple inverse scattering technique
}

\author{
L. Favro, X. Han, P. Kuo, R. Thomas
}

\section{To cite this version:}

L. Favro, X. Han, P. Kuo, R. Thomas. Improving the resolution of pulsed thermal wave images with a simple inverse scattering technique. Journal de Physique IV Proceedings, 1994, 04 (C7), pp.C7-545C7-550. 10.1051/jp4:19947128 . jpa-00253182

\section{HAL Id: jpa-00253182 https://hal.science/jpa-00253182}

Submitted on 1 Jan 1994

HAL is a multi-disciplinary open access archive for the deposit and dissemination of scientific research documents, whether they are published or not. The documents may come from teaching and research institutions in France or abroad, or from public or private research centers.
L'archive ouverte pluridisciplinaire HAL, est destinée au dépôt et à la diffusion de documents scientifiques de niveau recherche, publiés ou non, émanant des établissements d'enseignement et de recherche français ou étrangers, des laboratoires publics ou privés. 


\title{
Improving the resolution of pulsed thermal wave images with a simple inverse scattering technique
}

\author{
L.D. Favro, X. Han, P.K. Kuo and R.L. Thomas \\ Department of Physics and Institute for Manufacturing Research, Wayne State University, Detroit, MI \\ 48202, U.S.A.
}

\begin{abstract}
We have developed an inverse scattering technique for removing the blurring of pulse-echo thermal wave images. Preliminary results have been presented elsewhere [1-5]. We have extended the method to include thermally anisotropic materials and also multiple scattering effects. The method provides an algorithm for inverting a single image, and obtains the shape of the scatterer with a single calculation. The method involves no tomographic reconstruction and does not rely on a successive approximation scheme.
\end{abstract}

\section{INTRODUCTION}

It might be thought that solutions to the heat diffusion equation, which describes an irreversible process, would not be amenable to calculations which attempt to reverse the direction of time, and hence discover the sources of the heat after it has diffused away from those sources. It would seem even less likely that experimental temperature distribution data could be inverted to find the sources of the heat which produced those distributions. It is obvious that one cannot find the sources of the heat in a sample which has come to complete equilibrium, so that it is certainly true that one cannot, in general, invert a heat flow in time. However, there exists a fairly wide range of physical situations in which temperature distributions can be inverted, at least approximately, to find an earlier temperature distribution, and many of these situations are of considerable practical interest. In this paper we address such a situation: the case of a thermal wave image which results from a thermal wave pulse's propagation from the surface of a sample down to a planar subsurface defect, scattering from that defect, and subsequent propagation back to the surface of the sample. The mathematical model assumes that an image of the sample's surface temperature is made at some arbitrary instant of time after the signal from the scatterer has propagated back to the surface, and a single calculation is performed on that one image to produce an inverted image which shows the size and shape of the scatterer. Experimentally, the images are obtained by pulsed heating of the surface of the sample with an array of photographic flash lamps, followed by the formation of a time-gated image of the temperature distribution on that surface using an infrared video camera, all under the control of a PC computer. In the following sections we first describe a mathematical model for calculating the scattering of thermal waves, then describe the procedure for inverting that calculation, and finally show experimental results.

\section{MATHEMATICAL MODEL}

Although the inverse scattering technique will be applied only to planar scatterers, we will present the mathematical model for a more general scatterer because of its usefulness in direct scattering calculations where no inversion is needed. We imagine a scatterer of arbitrary shape imbedded in an semi-infinite sample with a plane surface, as indicated by $S_{3}$ in Fig. 1 . The sample surface $S_{1}$ is assumed to have 
been heated with an infinitely short, spatially uniform, pulse at $t=0$. The equation satisfied by the temperature in the sample (excluding the defect region) is then given by,

$$
\nabla^{\prime 2} T\left(\mathbf{r}^{\prime}, \mathbf{t}^{\prime}\right)=\frac{1}{\alpha} \frac{\partial}{\partial t^{\prime}} T\left(\mathbf{r}^{\prime}, \mathbf{t}^{\prime}\right)
$$

where $\alpha$ is the thermal diffusivity of the material, where the normal derivative of 'T( $\left.\mathbf{r}^{\prime}, \mathbf{t}^{\prime}\right)$ vanishes on the surface $S_{1}$, and where it is assumed that appropriate boundary conditions have been applied on the defect surface $S_{3}$.

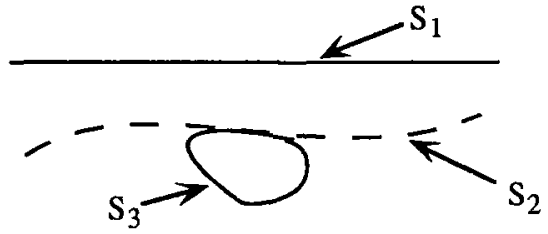

Fig. 1. Schematic diagram of the sample showing three surfaces. $S_{1}$ is the actual surface of the sample, $S_{2}$ is an imaginary surface used for purposes of integration, and $S_{3}$ is the surface of the defect.

The initial condition on the temperature distribution is specified by a limiting condition which specifies that,

$$
\mathrm{T}\left(\mathbf{r}, 0^{+}\right)=\mathrm{C} \delta\left(\mathbf{z}^{+}\right)
$$

where $\delta(\mathrm{z})$ is the Dirac delta function, where the superscript " + " indicates the limit as a variable approaches zero from the positive side, and where we have assumed that the inward normal to the sample's surface defines the positive z-direction. This condition corresponds to the sample's having been uniformly pulse heated on the surface $\mathrm{z}=0$ at a time $\mathrm{t}=0$. The constant $\mathrm{C}$ is determined by the amount heat per unit area deposited on the surface, and will hereafter be set equal to unity. A formal solution to this equation can be found by defining a Green's function which satisfies the equation,

$$
\nabla^{\prime 2} G\left(\mathbf{r}, \mathbf{r}^{\prime}, t-t^{\prime}\right)=-\frac{1}{\alpha} \frac{\partial}{\partial t^{\prime}} G\left(r, r^{\prime}, t-t^{\prime}\right)
$$

where two, and only two, conditions are imposed on $G\left(\mathbf{r}, \mathbf{r}^{\prime}, t-t^{\prime}\right)$. One is that, like $T\left(\mathbf{r}^{\prime}, t^{\prime}\right)$, its normal derivative also vanishes on $S_{1}$. The other is that, at $t=0$, it have the limiting behavior,

$$
\mathrm{G}\left(\mathbf{r}, \mathbf{r}^{\prime}, 0^{+}\right)=\delta\left(\mathbf{r}-\mathbf{r}^{\prime}\right)
$$

We should emphasize that we are not imposing any boundary conditions on the Green's function at the surface of the scatterer $S_{3}$. Although we later may want to impose some boundary conditions on the Green's function, at present, any function which satisfies Eqs. (2) and (3) is acceptable. If we now use Eqs. (1) and (2) in Green's theorem with an integration volume $V$ which is defined as the region between the surfaces $S_{1}$ and an arbitrary surface $S_{2}$, and integrate the result over $t$ ' from 0 to $t$, we obtain,

$$
\alpha \int_{0}^{t} d t^{\prime} \int_{S_{2}} n \cdot\left[G \nabla^{\prime} T-T \nabla^{\prime} G\right] d S^{\prime}=-\int_{V} d^{3} r^{\prime} G\left(r, r^{\prime}, t\right) T\left(r^{\prime}, 0^{+}\right)+\left\{\begin{array}{cc}
T(r, t) & r \in V \\
0 & r \notin V
\end{array}\right.
$$

where $\mathbf{n}$ is the outward normal to $\mathrm{V}$. It should also be noted that the last term in this equation is discontinuous across the boundary of the region $\mathrm{V}$. This discontinuous term arises from the delta function which results from the evaluation of the Green's function at $t^{\prime}=t$. The reason it is discontinuous is that 
the volume integration is only over the volume between the two surfaces, and hence does not include the entire volume of the sample. Since the delta function cannot contribute to the integral unless the region of integration includes its singularity, there is no contribution from it when $\mathbf{r}$ is outside of V. This discontinuity is compensated by a corresponding discontinuity in the surface integral on the left side of Eq. 4, but, as long as we are not interested in the temperature distribution outside of the region $\mathrm{V}$, we need not concern ourselves with the nature of the discontinuities, and they can be ignored. This will be the case throughout the rest of this paper. Now, using the methods of Ref. [4], it is easily shown that, if we let $T_{0}(r, t)$ represent the temperature that would result from a uniform heat pulse on the surface of a perfect sample, i.e. without the defect $\mathrm{S}_{3}$,

then

$$
T_{0}(r, t)=(\pi \alpha t)^{-1 / 2} \exp \left(-\frac{z^{2}}{4 \alpha t}\right)
$$

$$
\alpha \int_{0}^{t} d t^{\prime} \int_{S_{2}} n \cdot\left[G \nabla^{\prime} T_{o}-T_{o} \nabla^{\prime} G\right] d S^{\prime}=-\int_{V} d^{3} r^{\prime} G\left(r, r^{\prime}, t\right) T\left(r^{\prime}, 0^{+}\right)+T_{o}(r, t)
$$

This equation can be subtracted from Eq. 5 to obtain an equation for what might be called the "contrast temperature", i.e., the difference between the actual temperature $T$ (with the defect), and the temperature $\mathrm{T}_{\mathrm{o}}$ (without the defect),

$$
T(r, t)-T_{o}(r, t)=\alpha \int_{0}^{t} d t^{\prime} \int_{S_{2}} n \cdot\left[G \nabla^{\prime}\left(T-T_{0}\right)-\left(T-T_{0}\right) \nabla^{\prime} G\right] d S^{\prime}
$$

If one were clever enough to be able to guess the value of the temperature difference $\left(T-T_{0}\right)$ everywhere on the surface $S_{2}$, Eq. 8 would provide an expression for the temperature at all points in the volume $V$. In practice, this is impossible, except in trivial cases. Hence, to proceed further, one must be able to introduce some approximation scheme for evaluating the integral in the equation. The first step in approximating the integral is to decide what surface $S_{2}$ is to be used. This will depend on the nature of the defect under consideration, and one's ability to obtain an approximate expression on for the temperature on the chosen surface. One possibility suggests itself immediately, namely choosing $S_{2}$ to coincide with the defect's surface $S_{3}$. The usefulness of this choice depends on whether one can make a reasonable guess as to the degree to which the defect perturbs the temperature distribution on its surface. Another possibility is one in which the surfaces $S_{2}$ and $S_{3}$ are coincident over only part of each surface. This is the choice which is used in the inversion technique for planar scatterers to be described in the next section.

\section{INVERSION TECHNIQUE}

A very common application of thermal wave imaging is for the detection and sizing of delaminations and disbonds in coated or multi-ply materials. The defects of interest usually have a planar, or nearly planar, surface which is parallel, or nearly parallel, to the sample's front (heated) surface. In this situation it is convenient to choose the surface $S_{2}$ to be parallel to the front surface, and passing through the portion of $S_{3}$ which is parallel to the front surface. This is illustrated in Fig. 2, in which we have imagined $S_{3}$ to be

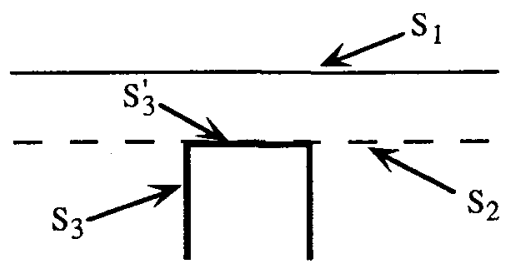

Fig. 2. The surfaces $S_{2}$ and $S_{3}$ may be partially or wholly coincident. In the illustration above, the defect is imagined to be a flat-bottomed hole with an arbitrarily shaped planar top surface. The surface $S_{2}$ is then taken to be the infinite extension of that planar surface. 
an arbitrarily shaped flat-bottomed hole milled in to the rear surface of the sample instead of a delamination. The reason for using a flat-bottomed hole as an illustration is to emphasize that the defect need not be totally planar. The surface $S_{2}$ can now be taken to be an infinite plane parallel to, and coincident with, the planar top surface of the flat-bottomed hole. The remaining problem is to find some approximate expression for the temperature difference $\left(T-T_{0}\right)$ on $S_{2}$. Perhaps the simplest approximation is the one which is almost always used in optical and acoustic diffraction calculations. In this approximation, the temperature field is taken to be the undisturbed incident thermal wave pulse, $T_{0}(r, t)$, on the region of $S_{2}$ away from $S_{3}$, and taken to be twice $T_{0}(r, t)$ on $S_{3}$. The reason for the factor of two on $S_{3}$ is that the reflected thermal wave from a void, in this case our flat-bottomed hole, is in phase with the incident wave and doubles it. We further require that the normal derivative of the temperature vanish on $S_{3}$, because at an air boundary the heat flux is essentially zero. As a result of these approximations, the integral over $S_{2}$ in Eq. 8 is reduced to an integral over the planar top surface of $S_{3}$, and Eq. 8 becomes,

$$
T(r, t)-T_{0}(r, t)=-\alpha \int_{0}^{t} d t^{\prime} \int_{S_{3}^{\prime}} n \cdot\left[G \nabla^{\prime} T_{o}+T_{o} \nabla^{\prime} G\right] d S^{\prime}
$$

where the prime on $S_{3}$ indicates that the integration is only over the top surface of the defect. In order to obtain an expression which can be inverted easily, we will choose a particularly simple form for the Green's function, namely the one which is ordinarily used for an infinite half-space,

$$
\mathrm{G}_{\mathbf{0}}\left(\mathbf{r}, \mathbf{r}^{\prime}, \mathrm{t}\right)=(4 \pi \alpha \mathrm{t})^{-3 / 2}\left[\exp \left(-\frac{\left(\mathbf{r}-\mathbf{r}^{\prime}\right)^{2}}{4 \alpha \mathrm{t}}\right)+\exp \left(-\frac{(\mathbf{r}-\widetilde{\mathbf{r}})^{2}}{4 \alpha t}\right)\right]
$$

With this choice of Green's function, the integration over time in Eq. 9 can be performed analytically, and a final expression for the contrast temperature thus obtained. That expression can be written in a more suggestive form by introducing what might be called a "scatterer shape function", which describes the (unknown) shape of the top surface of the defect, in the form,

$$
f\left(x^{\prime}, y^{\prime}\right)= \begin{cases}1 & \left(x^{\prime}, y^{\prime}\right) \in s_{3}^{\prime} \\ 0 & \left(x^{\prime}, y^{\prime}\right) \notin S_{3}^{\prime}\end{cases}
$$

The introduction of this shape function allows the spatial integrations in Eq. 9 to be formally extended to infinity, thus producing a final expression for the contrast temperature in the form of a two dimensional convolution integral,

$$
\begin{aligned}
& T(\mathbf{r}, \mathrm{t})-\mathrm{T}_{\mathrm{o}}(\mathrm{r}, \mathrm{t}) \cong \\
& -\frac{1}{2 \pi(\pi \alpha \mathrm{t})}{ }^{1 / 2} \frac{\partial}{\partial l} \int_{-\infty}^{\infty} \mathrm{d} \mathrm{x}^{\prime} \int_{-\infty}^{\infty} \mathrm{d} \mathrm{y}^{\prime} \frac{\exp \left[-\frac{\left\{\left[\left(x-x^{\prime}\right)^{2}+\left(y-y^{\prime}\right)^{2}+\mathrm{l}^{2}\right]^{1 / 2}+l^{2}\right\}^{2}}{4 \alpha t}\right]}{\left[\left(x-x^{\prime}\right)^{2}+\left(y-y^{\prime}\right)^{2}+l^{2}\right]^{1 / 2}} f\left(x^{\prime}, y^{\prime}\right)
\end{aligned}
$$

where, for simplicity, we have evaluated the expression for a point on the surface of the sample given by $(x, y, 0)$, and have assumed that the top surface of the defect is at a depth $l$ below the sample's surface. The fact that this approximate expression is a convolution of a known function with the unknown scatterer shape function, $f(x, y)$, immediately suggests a simple inversion technique. The Fourier transform of the convolution can be divided by the Fourier transform of the known function, yielding the transform of the shape function. An inverse Fourier transform should then produce the desired scatterer shape, $f(x, y)$. This technique fails to work in actual practice, because both detector noise and digitization noise (the experimental images are digitized at the 8-bit level), obscure the image signal. However, a simple low- 
pass filtering operation, which removes the higher spatial frequencies from the Fourier transform of the image, suffices to restore most of the features of the shape of the defect in the inverted image. [1-5]

It is possible to improve the accuracy of the approximation in Eq. 12 by making more realistic defect models, by choosing better approximations to the temperature field on the surface of the defect, and by more sophisticated choices of the form of the Green's function. Detailed derivations of such formulae will be published elsewhere, but one will be exhibited here to illustrate the kind of problems which can be handled with the technique. Imagine that the flat-bottomed hole in the example above were filled with some other material so that $S_{3}$ could no longer be approximated as an insulated surface. Imagine further that the material of the sample were anisotropic, and that the top of the defect were so close to the sample's surface that the thermal wave pulse would undergo several reflections back and forth between the two surfaces before it decayed away. Under these circumstances the approximations leading to Eq. 12 are no longer appropriate. However, by modifying the Green's function and/or the boundary conditions on $S_{3}$, all of these imagined changes can be accommodated. In order to invert the experimental image to be shown below, we will need an approximation which takes account of both multiple reflections and anisotropy. This can be accomplished by changing the Green's function to one which contains an infinite series of terms which represent the back and forth reflections of the thermal wave pulse. This procedure outlined above can again be carried out to produce an expression for the contrast temperature in the form of a convolution to a known function with the scatterer shape function,

$$
\mathrm{T}-\mathrm{T}_{\mathrm{o}}=\frac{1}{2 \pi}\left(\frac{1}{\pi \alpha_{1} \alpha_{2} \alpha_{3} \mathrm{t}}\right) \int_{-\infty}^{1 / 2} \int_{-\infty}^{\infty} \mathrm{dx} \mathrm{dy}^{\prime} \sum_{\mathrm{m}=1}^{\infty} \frac{\mathrm{A}^{\mathrm{m}}}{\mathrm{R}_{\mathrm{m}}} \frac{1}{\alpha_{3}^{1 / 2}}\left[\frac{\partial}{\partial \mathrm{z}} \exp \left\{-\frac{\left[\mathrm{R}_{\mathrm{m}}+\left(\mathrm{z}^{2} / \alpha_{3}\right)^{1 / 2}\right]^{2}}{4 \mathrm{t}}\right\}\right]_{\mathrm{z}=\mathrm{l}} \mathrm{f}\left(\mathrm{x}^{\prime}, \mathrm{y}^{\prime}\right)
$$

where

$$
R_{m}=\left[\frac{\left(x-x^{\prime}\right)^{2}}{\alpha_{1}}+\frac{\left(y-y^{\prime}\right)^{2}}{\alpha_{2}}+\frac{(2 m+1)^{2} \ell^{2}}{\alpha_{3}}\right]^{1 / 2}
$$

where $\alpha_{1}, \alpha_{2}$, and $\alpha_{3}$ are the diffusivities along the principle axes of the diffusion tensor (with the $z$-axis assumed to be aligned with the normal to the sample), and where $A$ is the thermal wave reflection coefficient at the surface of the defect.
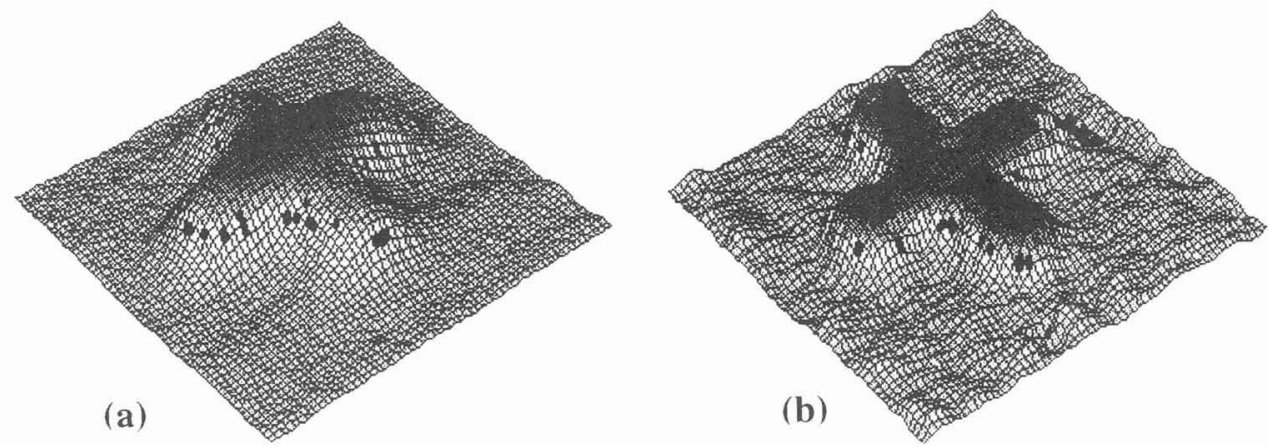

Fig. 3 (a) A raw thermal wave image of a cross-shaped flat-bottomed hole in an anisotropic graphite-epoxy sample. The high conductivity direction is from the lower left to the upper right. (b) The inverted image showing the improved resolution and flat top surface of the cross. 
In order to test the inversion technique under more rigorous conditions, we have prepared a graphite epoxy sample with uniaxial fibers running parallel to the surface. The fibers provide a thermal conductivity which is an order of magnitude higher in the direction of the fibers than in the two directions perpendicular to the fibers. In the rear of this sample we milled a flat-bottomed hole in the shape of a cross, with the top surface of the hole close enough to the surface of the sample to cause multiple thermal-wave reflections to be important. Figures $3 \mathrm{a}$ and $3 \mathrm{~b}$, respectively, show the original experimental thermal-wave image and its inversion for this sample. The first six terms in the series of Eq. 13 contributed significantly to the image. It can be seen by comparison of Figs. 3 (a) and 3 (b) that the inversion has substantially restored the flattopped shape of the cross producing a significant improvement in the resolution of the edges. It may also be noted that, in spite of the low-pass spatial filtering used in the inversion, additional structure is found in the inverted image, away from the cross. This corresponds to the presence of porosity which was obscured in the original, blurred, thermal wave image.

\section{Acknowledgments}

This work was sponsored by the Institute for Manufacturing Research, Wayne State University.

\section{References}

[1] Favro L.D., Crowther D., Kuo P.K., and Thomas R.L., Proc. SPIE Thermosense XIV Orlando, FL, April 20-24, 1992, Vol. 1682 Thermosense XIV (1992), 178-181.

[2] Crowther D., Favro L.D., Kuo P.K., and Thomas R.L., Photoacoustic and Photothermal Phenomena III, D. Bicanic (Ed.) Springer Series in Optical Sciences 69, 544-546, SpringerVerlag, Berlin, Heidelberg (1992).

[3] Favro L.D., Crowther D., Kuo P.K., and Thomas R.L., Proc. 14th International Congress on Acoustics, Beijing, Sept. 3-10, 1992, A13-5.

[4] Crowther D., Favro L.D., Kuo P.K., and Thomas R.L., J. Appl. Phys. 74 (9) 5828-5834, (1993).

[5] Favro L.D., Crowther D., Kuo P.K., and Thomas R.L., Proc. SPIE Thermosense XV , Vol. 1993, pp. 138-141 (1993). 